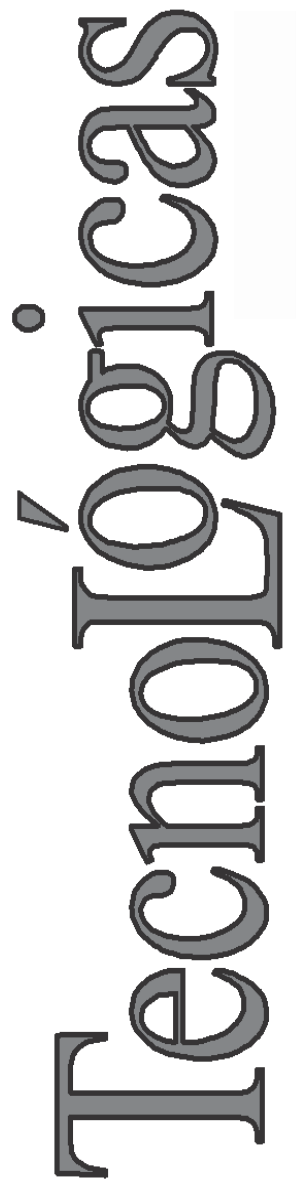

\title{
Identificación de un Motor de Corriente Directa de Imanes Permanentes a Partir de un Único Experimento
}

\section{Identification of a Permanent Magnet Direct Current Motor from One Experiment}

Juan C. González ${ }^{1}$ Andrés J. Saavedra-Montes ${ }^{2}$ Carlos A. Ramos-Paja ${ }^{3}$

1 Fluxcontrol SAS, Medellín-Colombia jgonzalez@fluxcontrol.com.co 2 Departamento de Energía Eléctrica y Automática, Facultad de Minas, Universidad Nacional de Colombia, Medellín-Colombia ajsaaved@unal.edu.co 3 Departamento de Energía Eléctrica y Automática, Facultad de Minas, Universidad Nacional de Colombia, Medellín-Colombia caramosp@unal.edu.co 


\section{Resumen}

En este artículo se presenta un procedimiento simple para estimar los parámetros de las funciones de transferencia, basándose en la respuesta al escalón, de un sistema conformado por un driver de velocidad y un motor de corriente directa de imanes permanentes. El proceso de identificación consiste en la selección de los modelos, la adquisición y preprocesamiento de los datos, la estimación de parámetros y su validación. Los resultados muestran que es posible estimar los parámetros del sistema a partir de la respuesta al escalón, logrando valores de la suma normalizada de los errores cuadráticos menores al 0,5\%.

\section{Palabras clave}

Motor de corriente directa; driver de velocidad; modelo dinámico; estimación de parámetros, respuesta al escalón.

\section{Abstract}

A simple procedure to estimate the transfer function parameters, based on the step response, of a system composed by a speed driver and a permanent magnet direct current motor is presented in this paper. The identification process is composed by the model selection, the acquisition and pre-processing of data, the parameter estimation and its validation. The results show that it is possible to estimate the system parameters from the step response achieving values of the normalized sum of squared errors less than $0.5 \%$.

\section{Keywords}

Direct current motor; speed driver; dynamic model; parameter estimation; step response. 


\section{INTRODUCCIÓN}

Los motores de corriente directa excitados con imanes permanentes (MCDIP) se están utilizando actualmente en un rango de potencias que van desde los $\mathrm{mW}$ hasta los MW (Gieras \& Wing, 2002), sin embargo se reconocen principalmente en las aplicaciones con potencias menores a $1 \mathrm{~kW}$.

Las múltiples aplicaciones de los MCDIP requieren alguna variable controlada, ya sea la velocidad del motor, el par de salida, etc. Por lo tanto, los MCDIP se integran a sistemas de control que típicamente están compuestos por un controlador, un convertidor de potencia, el MCDIP y uno o más sensores. El diseño de los controladores de velocidad para motores de corriente directa se basa en la respuesta transitoria del motor, despreciando las dinámicas del actuador y del sensor. Esta aproximación tiene menor efecto, en el diseño del controlador, en la medida que las dinámicas del convertidor y el sensor sean menores a la dinámica del motor. Sin embargo, existen casos en los cuales el tiempo de respuesta del convertidor es comparable con el tiempo de respuesta del motor, requiriendo que ambas dinámicas sean consideras en el diseño del controlador de velocidad. Por lo tanto, para controlar este tipo de sistemas, se necesitan valores confiables de los parámetros de los modelos matemáticos que describen el comportamiento del motor y de los actuadores.

Considerando lo anterior, en (Tutunji et al., 2007) se presenta un algoritmo de identificación recursivo para identificar sistemas mecatrónicos: un motor de corriente directa y un giroscopio. Se utiliza una tensión de entrada tipo impulso para excitar el sistema y se mide la salida de velocidad angular. En dicho documento se realiza una validación estadística de los modelos y para identificar los parámetros se realizan veintiséis experimentos.

De forma simular, en (Kapun et al., 2008) se presenta un método de identificación fuera de línea basado en la técnica de aproximación de mínimos cuadrados, donde se aplica un observador de perturbación de lazo cerrado para la identificación de los parámetros del modelo de un MCDIP. Éste método se utiliza para identificar la fuerza contra-electromotriz y los parámetros mecánicos. En este documento se realizan cuarenta experimentos para determi- 
nar los parámetros del modelo, se validan los parámetros del modelo de forma estadística, se hace la estimación de algunos de los parámetros del modelo de forma independiente y además, no se comparan las salidas del modelo real y el modelo simulado.

En este artículo se presenta la identificación de los parámetros de un motor de corriente directa y su driver de velocidad, usando un único experimento. Con este fin, se seleccionan los modelos tanto para el motor de corriente directa como para su driver de velocidad, luego se diseña y se lleva a cabo el experimento para la identificación de los parámetros. Posteriormente, se pre-procesan los datos obtenidos en el experimento y se aplica el algoritmo de identificación de parámetros. Los resultados obtenidos se validan comparando las salidas del sistema simulado y el sistema real por medio de un índice de error. Finalmente, la principal contribución del procedimiento propuesto consiste en requerir un único experimento para determinar todos los parámetros del motor y del driver de potencia.

\section{MODELO DE UN MOTOR DE CORRIENTE DIRECTA Y SU DRIVER DE VELOCIDAD}

La identificación de parámetros se aplicó al sistema compuesto por un driver de velocidad ajustable (DVA) y un MCDIP. En la industria existen procesos en los que se requiere ajustar y controlar la velocidad, posición o torque de un motor, ya sea para su funcionamiento nominal o para ajustarse a los requerimientos de un proceso. Estas operaciones se realizan con DVAs. Generalmente, un DVA está compuesto por una etapa de potencia y otra etapa de control, ver Fig. 1. Los DVA requieren medir algunas variables eléctricas y mecánicas para realizar el control y la protección del motor eléctrico (Rahman et al., 2007): Se miden las corrientes de entrada $i_{i}$ y salida $i_{o}$, los estados del convertidor de potencia x y la velocidad del motor $\omega$. El sistema de control, por su parte, genera las señales de desconexión de la línea AC y la señal de control u de los semiconductores. 


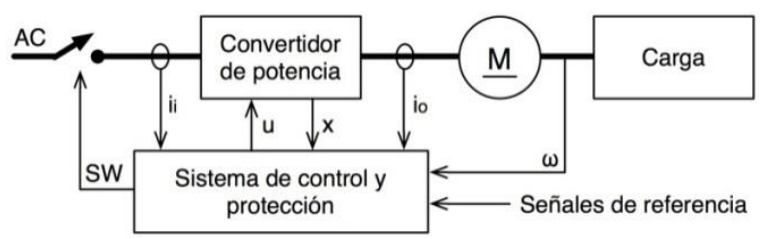

Fig. 1. Diagrama de bloques de un DVA acoplado a un motor eléctrico. Fuente: Autores

El DVA utilizado en este trabajo es el SCR DC MOTOR CONTROL BC142-5. Este dispositivo está compuesto por un rectificador de onda completa formado por cuatro rectificadores controlados de silicio (SCR). En este driver el voltaje de salida está dado por (1), donde $V_{m}$ corresponde al valor pico del voltaje de línea de entrada $V_{a c}=V_{m} \sin (\omega \mathrm{t}), V_{a}$ es el voltaje de armadura del motor CD y corresponde al voltaje de salida del driver. El valor de $V_{a}$ es controlado por medio del ángulo de disparo a (Rahman et al., 2007).

$V_{a}=\left(2 \cdot V_{m} / \pi\right) \cdot \cos (\alpha)$

Adicional al circuito rectificador, el DVA también está compuesto por circuitos de control y protección para el motor. El DVA BC142-5 permite ajustar la velocidad angular del motor $\left(\omega_{m}\right)$ a través del voltaje de armadura, donde $V_{a}$ se ajusta por medio de un voltaje de referencia $\left(V_{r e f}\right)$. Los límites que impone el driver

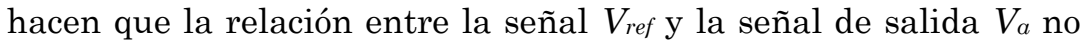
sea lineal, sin embargo en (Guinee, 2000) se propone una aproximación lineal para relacionar estas dos variables (2).

$\frac{V_{a}(s)}{V_{r e f}(s)}=\frac{H_{o} \omega_{o}^{2}}{s^{2}+2 \xi \omega_{o} s+\omega_{o}^{2}}$

El modelo del driver se considera en el proceso de identificación de parámetros, pues su dinámica altera el desempeño del lazo de control de velocidad del motor.

El MCDIP está formado por dos partes principales: El rotor o armadura y el estator. El estator consiste en imanes permanentes, los cuales crean un campo magnético. La armadura consiste de un 
electroimán creado por una bobina enrollada alrededor de un núcleo de hierro. La armadura rota debido al fenómeno de atracción y oposición de fuerzas de los dos campos magnéticos. Un campo magnético es generado por la armadura al hacer circular una corriente eléctrica a través de la bobina, el sentido de la corriente se intercambia constantemente para alternar la polaridad en el electroimán y hacer siempre coincidir los polos para generar la rotación de la armadura.

Las características eléctricas y mecánicas de un MCDIP se describen por (3)-(6) (Rahman et al., 2007). La Fig. 2 presenta un esquema equivalente del motor dc de imanes permanentes, describiendo las variables del modelo.

$$
\begin{aligned}
& V_{a}(t)=R_{a} i_{a}(t)+L_{a} \frac{d i_{a}(t)}{d t}+e(t) \\
& e(t)=k_{e} \omega_{m}(t) \\
& \tau_{e}(t)=j_{m} \frac{d \omega(t)}{d t}+B_{m} \omega_{m}(t)+\tau_{m} \\
& \tau_{e}(t)=k_{m} i_{a}(t)
\end{aligned}
$$

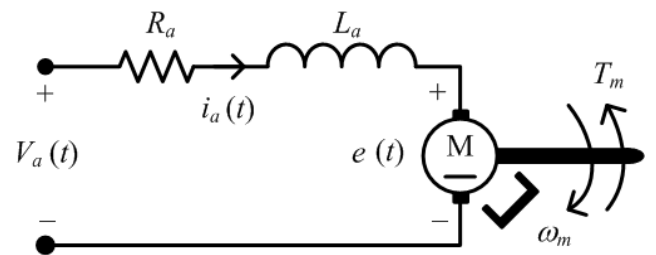

Fig. 2. Circuito equivalente de un motor DC de imanes permanentes. Fuente: Autores

Las ecuaciones diferenciales que representan el motor se obtienen reemplazando (4) en (3) y (6) en (5):

$$
\frac{d i_{a}(t)}{d t}=\frac{V_{a}(t)-R_{a} i_{a}(t)-k_{e} \omega_{m}}{L_{a}}
$$


$\frac{d \omega(t)}{d t}=\frac{k_{m} i_{a}(t)-B_{m} \omega_{m}(t)-\tau_{m}}{j_{m}}$

A partir de (7) y (8) se obtiene la representación del sistema en el espacio de estados descrita en (9), donde el vector de estados $x$, los jacobianos $A$ y $B$, y las matrices de la ecuación de salida $C$ y $D$ se presentan en (10).

$\dot{x}=A x+B u, y=C x+D u$

$x=\left[\begin{array}{c}i_{a} \\ \omega\end{array}\right], A=\left[\begin{array}{cc}-R_{a} / L_{a} & -k_{e} / L_{a} \\ k_{m} / j_{m} & -B_{m} / j_{m}\end{array}\right], B=\left[\begin{array}{cc}1 / L_{a} & 0 \\ 0 & -1 / j_{m}\end{array}\right]$,

$C=\left[\begin{array}{ll}0 & 1\end{array}\right], D=[0]$

A partir de la representación en espacio de estados se obtienen las funciones de transferencia (11)-(14), donde el valor de $B_{m}$ afecta poco el comportamiento del MCDIP y por tanto se desprecia. Asimismo, se considera $k_{e}=k_{m}=k$ y las constantes del denominador son $p_{1}=j_{m} L_{a}, p_{2}=j_{m} R_{a}$ у $p_{3}=k^{2}$.

$\frac{\omega_{m}(s)}{V_{a}(s)}=\frac{k}{p_{1} s^{2}+p_{2} s+p_{3}}$

$\frac{I_{a}(s)}{V_{a}(s)}=\frac{j_{m} s}{p_{1} s^{2}+p_{2} s+p_{3}}$

$\frac{\omega_{m}(s)}{\tau_{m}(s)}=-\frac{L_{a} s+R_{a}}{p_{1} s^{2}+p_{2} s+p_{3}}$

$\frac{I_{a}(s)}{\tau_{m}(s)}=\frac{k}{p_{1} s^{2}+p_{2} s+p_{3}}$

\section{ESTIMACIÓN DE PARÁMETROS DEL MODELO DEL MCDIP}

La metodología para la estimación de parámetros consiste de cuatro etapas. En la primera etapa se seleccionan los modelos matemáticos para el DVA y para el MCDIP. En la segunda etapa 
se diseña y ejecuta el experimento para la recolección de los datos requeridos por los procesos de la estimación y validación. En la tercera etapa se realiza el pre-procesamiento de los datos recolectados y se ejecuta el proceso de estimación de parámetros. Por último, los modelos y sus respectivos parámetros son validados.

\subsection{Selección de Modelos}

Los modelos matemáticos seleccionados para el DVA y el MCDIP se discutieron en la Sección 2: Para el DVA se seleccionó (2) y para el motor se seleccionaron (11) y (12).

\subsection{Experimento para Recolección de Datos}

La recolección de los datos utilizados para los procesos de estimación y validación se llevó a cabo mediante un experimento en el que se utilizó un MCDIP, un DVA, un osciloscopio digital, una fuente de voltaje programable e instrumentación adicional, ver Fig. 3.

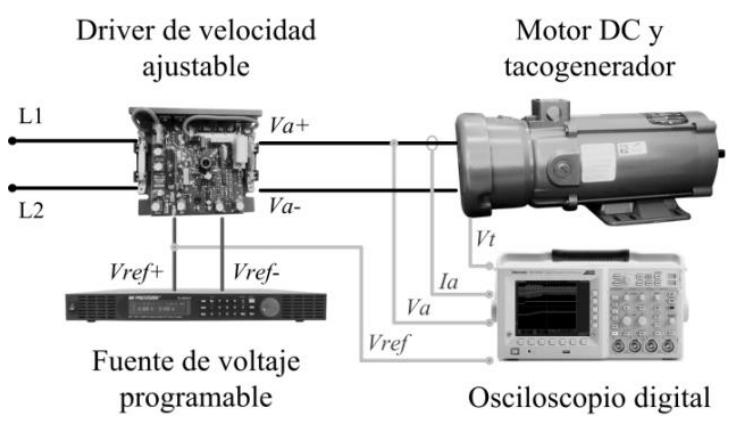

Fig. 3. Configuración de los equipos de laboratorio. Fuente: Autores

Se seleccionó un motor Baldor CDPT3455 para la estimación de sus parámetros, el motor tiene una potencia nominal de $746 \mathrm{~W}$, un voltaje de armadura de $180 \mathrm{~V}$, una corriente de armadura de $5 \mathrm{~A}$ y una velocidad nominal de 1750rpm. Éste motor cuenta además con un tacogerenerador para la medición indirecta de la velocidad a través de un voltaje $V_{\text {taco }}$. Asimismo, se utilizó un DVA Baldor $B C$ 142-5, el cual se alimenta a $220 \mathrm{~V}$ CA y suministra un voltaje 
de armadura $V_{a}$ entre $0 \mathrm{~V}$ y $180 \mathrm{~V}$. El voltaje $V_{a}$ se obtiene a través de un voltaje de referencia $V_{\text {ref }}$ entre $0 \mathrm{~V}$ y $9 \mathrm{~V}$. Para manipular $V_{\text {ref }}$ se utilizó la fuente de voltaje programable BK Precision XLN6024, la cual permite generar señales de excitación tipo escalón. El osciloscopio Tectronix TDS 3034c posibilitó la captura simultánea de cuatro variables.

El experimento realizado para la recolección de datos consistió en obtener la respuesta del DVA y del MCDIP ante dos señales tipo escalón de diferente magnitud: La primera señal se utilizó para generar los datos requeridos para la estimación de los parámetros y la segunda se utilizó para generar los datos de validación. Las respuestas a señales tipo escalón son ampliamente usadas para la identificación de parámetros en sistemas mecánicos, electromecánicos y químicos, entre otros, ya que permiten excitar el sistema en un rango amplio de frecuencias. Este tipo de señales también permiten obtener diferentes características de los sistemas como sobrepaso, tiempo de estabilización y tiempo de subida (Kuo, 1996).

En el experimento se aplican los dos escalones de voltaje de forma consecutiva: El primer escalón impone un cambio de voltaje de referencia del DVA entre $7 \mathrm{~V}$ a $9 \mathrm{~V}$ en el voltaje, generando un cambio aproximado de $140 \mathrm{~V}$ a $180 \mathrm{~V}$ en $V_{a}$; este escalón se nombró "segmento de gran señal". El segundo escalón impone un cambio de voltaje de referencia del DVA entre $8.5 \mathrm{~V}$ a $9 \mathrm{~V}$, generando un cambio aproximado de $170 \mathrm{~V}$ a $180 \mathrm{~V}$ en $V_{a}$; este escalón se nombró "segmento de pequeña señal". Los datos del segmento de gran señal se utilizaron para el proceso de estimación, y los datos del segmento de pequeña señal se usaron en el proceso de validación.

En el experimento se capturaron los datos de $V_{r e f}, V_{a}, V_{\text {taco }}$ e $I_{a}$ utilizando el osciloscopio a una frecuencia de $5000 \mathrm{~Hz}$, ver Fig. 4. Para obtener el valor la velocidad angular en $[\mathrm{rad} / \mathrm{s}]$ se usa $\omega_{m}=$ $2.094 V_{\text {taco, }}$ obtenida desde la ganancia del tacogenerador.

\subsection{Pre-procesamiento de los Datos}

El pre-procesamiento de los datos se hace con el fin de remover la información que no corresponde al sistema y que afecta el proceso de estimación, e.g. ruido introducido en la medición y datos 
atípicos. El pre-procesamiento para los datos de estimación y validación consistió en aplicar a las diferentes señales de $V_{r e f}, V_{a}$, $\omega_{m}$ e $I_{a}$ un filtro de segundo orden tipo Butterworth con frecuencia de corte igual a $70 \mathrm{rad} / \mathrm{s}$. Ésta frecuencia de corte se determinó con base en el tiempo de respuesta del sistema. Posteriormente, se retiró el valor medio de las señales. Las señales experimentales y pre-procesadas se observan en la Fig. 5.

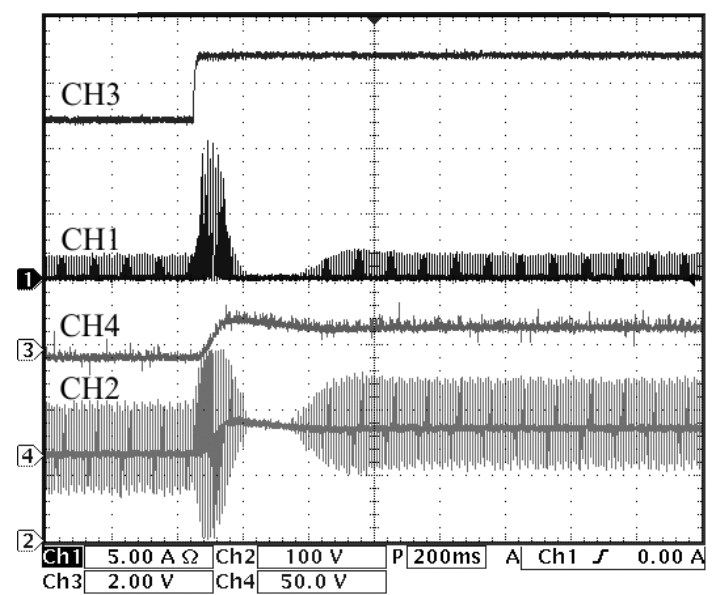

Fig. 4. Señales capturadas; Ia $(\mathrm{CH} 1), V_{a}(\mathrm{CH} 2), V_{\text {ref }}(\mathrm{CH} 3), V_{\text {taco }}(\mathrm{CH} 4)$.

Fuente: Autores
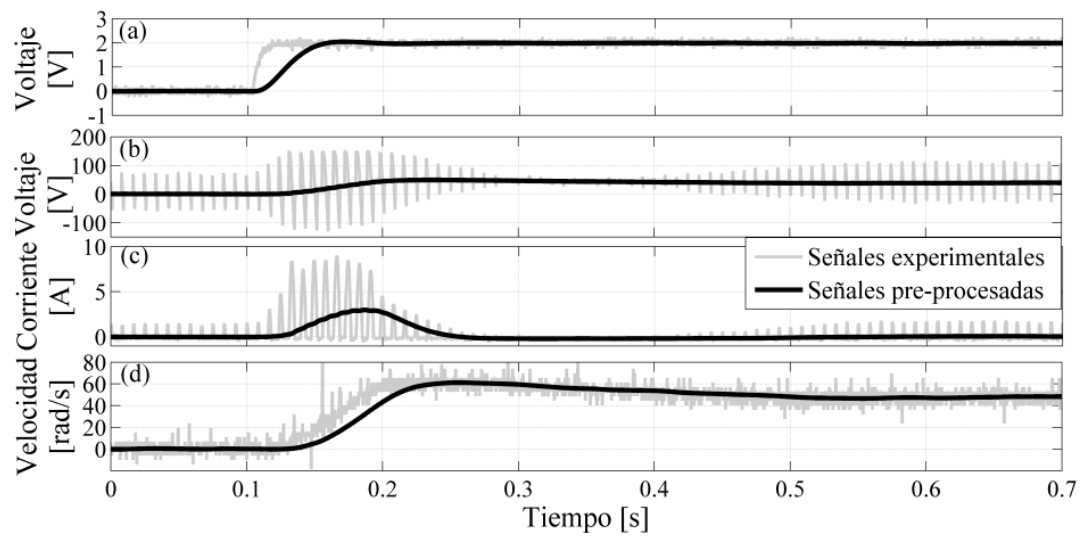

Fig. 5. Señales experimentales y pre-procesadas: (a) $V_{r e f}$, (b) $V_{a}$, (c) $I_{a}$, (d) $\omega_{m}$. Fuente: Autores 
Posterior al pre-procesamiento de las señales se realizó la estimación de parámetros de las funciones de transferencia descritas en (11) y (12), es decir, las funciones de transferencia cuya entrada es $V_{a}$. Para las funciones de transferencia descritas en (13) y (14) su entrada $\tau_{m}$ es desconocida por lo que no se incluyen en la estimación de parámetros. La estimación de parámetros se realizó utilizando la herramienta Parameter Estimation de Simulink/Matlab. Ésta herramienta compara los datos experimentales con los datos generados por el modelo implementado en Simulink, usando técnicas de optimización, para estimar los parámetros a través de la reducción de una función de costo. Opcionalmente, el usuario puede ingresar las condiciones iniciales de los estados del modelo. La función de costo normalmente calcula el error cuadrático medio entre los datos correspondientes a las señales experimentales y a las señales estimadas.

\subsection{Validación del Modelo del MCDIP}

Los parámetros estimados del modelo (2) del DVA son $H_{o}=$ $20.177[-], \omega_{o}=35.460[\mathrm{rad} / \mathrm{s}]$ y $\xi=0.447[-]$. Los parámetros estimados para el MCDIP son $k=0.8022, j_{m}=2.0768 \cdot 10^{-3}, p_{1}=8.454$. $10^{-5}, p_{2}=0.0114$ y $p_{3}=0.6435$. A partir de dichos valores se obtienen los parámetros estimados para el motor DC reportados en la Tabla 1. La Tabla 1 también presenta los valores de referencia de $R_{a}, k, L_{a}$ y $J$, así como los errores en los parámetros estimados.

En (Saavedra-Montes et al., 2011) se propone la suma normalizada de los errores cuadráticos (SNEC) como índice de desempeño para comparar los datos de las salidas experimentales contra los datos de las salidas estimadas por un modelo. La ecuación (15) presenta la SNEC, donde $n$ es la longitud del conjunto de datos, $y_{i}$ son los datos correspondientes a la salida experimental y $\hat{y}_{i}$ son los datos correspondientes a la salida estimada.

$S N E C=100\left(\sum_{i=1}^{n}\left(y_{i}-\widehat{y_{l}}\right)^{2} / \sum_{i=1}^{n} y_{i}^{2}\right)$ 
Tabla 1. Comparación de los Parámetros de Referencia y Estimados. Fuente: Autores

\begin{tabular}{lccc}
\hline \multicolumn{1}{c}{ Parámetro } & Valor de Referencia & Valor Estimado & Error (\%) \\
\hline$k[\mathrm{~V} / \mathrm{rad} / \mathrm{s}]$ & 0.8071 & 0.8022 & 0.60 \\
$L_{a}[\mathrm{mH}]$ & 29.7340 & 31.233 & 5.04 \\
$R_{a}[\Omega]$ & 4.203 & 4.212 & 0.20 \\
$J_{a}\left[\mathrm{kgm}^{2}\right]$ & & $2.7068 \times 10^{-3}$ & \\
\hline
\end{tabular}

Los datos experimentales corresponden al segmento de pequeña señal luego de ser pre-procesados. Las señales estimadas fueron obtenidas por simulación usando los modelos seleccionados, con sus respectivos parámetros estimados, y la señal experimental de excitación perteneciente al segmento de pequeña señal.

La Fig. 6 presenta los resultados obtenidos en el proceso de validación, donde la señal de entrada corresponde al voltaje de referencia (a). En (b) se presenta la comparación entre la señal experimental y estimada para el voltaje de armadura, donde se obtuvo SNEC $=0.4526 \%$. En (c) y (d) se presenta la misma comparación para la corriente de armadura y la velocidad rotacional, respectivamente, donde para la corriente de armadura se obtuvo SNEC $=11.74 \%$ y para la velocidad rotacional se obtuvo $\mathrm{SNEC}=0.1141$ $\%$.

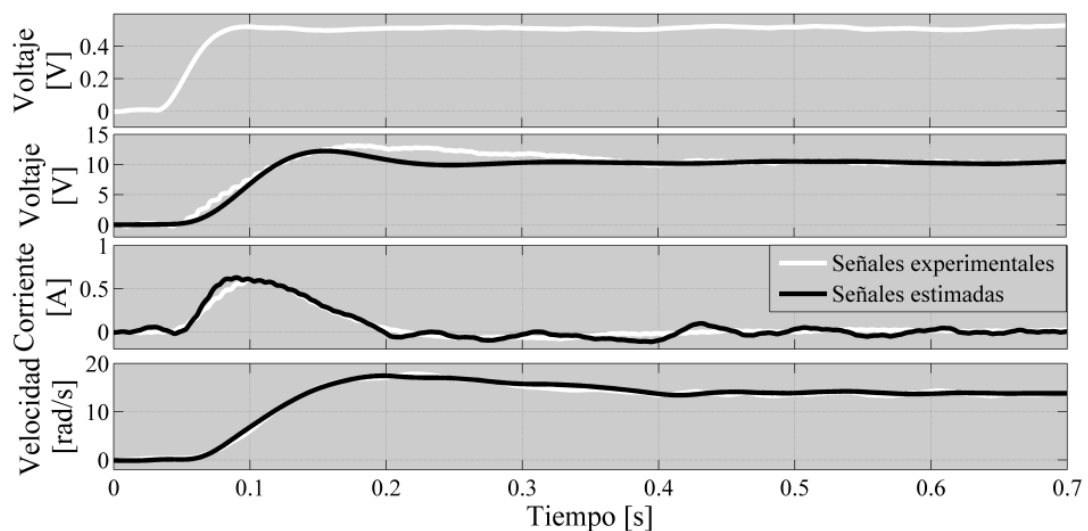

Fig. 6. Validación: (a) $V_{r e f}$, (b) $V_{a}$ vs $\widehat{V_{a}}$, (c) $I_{a}$ vs $\widehat{I}_{a}$, (d) $\omega_{m}$ vs $\widehat{\omega_{m}}$. Fuente: Autores 


\section{CONCLUSIONES}

Se presentó una metodología para estimar los parámetros de un motor DC y su driver de velocidad. La metodología tiene la ventaja de ser bastante sencilla, pues solo necesita una prueba llamada de pequeña señal para estimar los parámetros tanto del motor DC como del driver de velocidad. También se hallaron valores de referencia de los parámetros de la maquina con el fin de ser usados en la validación del modelo del motor DC. Los resultados muestran un error máximo en la comparación de parámetros con respecto a los parámetros de referencia por debajo de 6\%. Adicionalmente un resultado de la suma normalizada de errores al cuadrado menor de $0.5 \%$ para la comparación de las salidas del sistema real y el sistema simulado con los parámetros hallados por medio de la metodología usada ente la misma señal de entrada. Finalmente, los modelos obtenidos se usaron para hallar un controlador para el sistema en lazo cerrado con el fin de mostrar la utilidad de los modelos en el diseño de sistemas de control.

\section{AGRADECIMIENTOS}

Este trabajo fue soportado por la beca doctoral 095-2005 de COLCIENCIAS y por la Universidad Nacional de Colombia a través de los proyectos IDENT-EXGEN y SMART- ALEN.

\section{REFERENCIAS}

Gieras, J. F. \& Wing, M. (2002). Permanent magnet motor technology: design and applications. Marcel Dekker Inc. cement of Medical Instrumentation, 32(3), 241-52.

Guinee, R. A. (2000). Variable speed motor drive testing and parameter identification using pseudorandom binary sequences. In 10th Mediterranean Electrotechnical Conference (Vol. 3, pp. 1085-1088).

Kapun, A., Čurkovič, M., Hace, A. \& Jezernik, K. (2008). Identifying dynamic model parameters of a BLDC motor. Simulation Modelling Practice and Theory, 16(9), 1254-1265.

Kuo, B. C. (1996). Sistemas de control automático. Prentice Hall Hispanoamericana. 
Rahman, M. F., Patterson, D., Cheok, A. \& Betz, R. (2007). Chapter 33 Motor drives. In Power Electronics Handbook (Second Edition). Burlington Academic Press, 857-933.

Saavedra-Montes, A. J., Ramirez-Scarpetta, J. M. \& Malik, O. P. (2011). Methodology to estimate parameters of an excitation system based on experimental conditions. Electric Power Systems Research, 81(1), $170-176$.

Tutunji, T., Molhim, M. \& Turki, E. (2007). Mechatronic systems identification using an impulse response recursive algorithm. Simulation Modelling Practice and Theory, 15(8), 970-988. 